\title{
Leishmania infection and neuroinflammation: Specific chemokine profile and absence of parasites in the brain of naturally-infected dogs
}

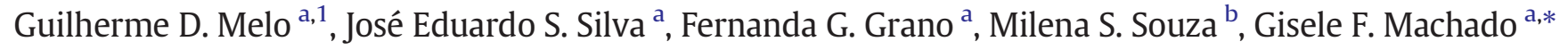 \\ a Faculdade de Medicina Veterinária, UNESP - Univ Estadual Paulista, Laboratório de Patologia Aplicada (LApap), Araçatuba, São Paulo, Brazil \\ b Faculdade de Medicina Veterinária, UNESP - Univ Estadual Paulista, Araçatuba, São Paulo, Brazil
}

\section{A R T I C L E I N F O}

\section{Article history:}

Received 24 June 2015

Received in revised form 28 August 2015

Accepted 8 October 2015

\section{Keywords:}

Chemokine CCL3

Chemokine CCL4

Chemokine CCL5

Central nervous system

T lymphocytes

Visceral leishmaniasis

\begin{abstract}
A B S T R A C T
Visceral leishmaniasis is a chronic disease caused by Leishmania infantum. We aimed to detect the parasite in the brain of fifteen naturally-infected dogs using in situ hybridization and immunohistochemistry, and the gene expression of selected chemokines by RT-qPCR. We detected no parasite in the brain, but perivascular deposition of parasite DNA and IgG in the choroid plexus. We noticed up-regulation of CCL-3, CCL-4 and CCL-5, coherent with T lymphocyte accumulation, stating the brain as a pro-inflammatory environment. Indeed, not necessarily the parasite itself, but rather its DNA seems to act as a trigger to promote brain inflammation during visceral leishmaniasis.
\end{abstract}

(c) 2015 Elsevier B.V. All rights reserved.

\section{Introduction}

Visceral leishmaniasis (VL) is a chronic disease caused by parasitic protozoans from the Leishmania donovani complex, namely $L$. (L.) donovani and Leishmania (L.) infantum ( syn = chagasi), which belong to the family Trypanosomatidae. Dogs are considered the main urban reservoir of this neglected disease, which presents worldwide distribution, and a zoonotic importance in Brazil and in the Mediterranean basin (Baneth et al., 2008; Chappuis et al., 2007).

Infected dogs present with different patterns of immune response against the parasite, with an effective cellular activation or a deleterious humoral response (Barbiéri, 2006). Infected dogs may keep asymptomatic for long periods or quickly develop the classical symptoms of the disease such as skin and ocular diseases, renal failure, anemia, cachexia and generalized lymphadenopathy (Alvar et al., 2004). Despite the predilection for the liver, spleen and bone marrow, the parasite could virtually spread everywhere, including the genital system (Diniz et al., 2005), muscles (Gomes et al., 2012), and the central nervous system (Márquez et al., 2013).

\footnotetext{
* Corresponding author at: Departamento de Clínica, Cirurgia e Reprodução Animal, Faculdade de Medicina Veterinária, Universidade Estadual Paulista "Júlio de Mesquita Filho" (UNESP), Rua Clóvis Pestana, 793, Araçatuba, São Paulo CEP 16050-680, Brazil.

E-mail addresses: gdiasdem@pasteur.fr (G.D. Melo), giselem@fmva.unesp.br (G.F. Machado).

1 Present address: Laboratoire des Processus Infectieux à Trypanosomatidés, Département Infection et Epidemiologie, Institut Pasteur, Paris, France.
}

Specifically in the brain, the parasite is not often detected (Márquez et al., 2013; Viñuelas et al., 2001), however, inflammatory lesions even in the absence of the parasite are commonly observed, predominantly leptomeningitis and choroitis, with accumulation of mononuclear cells (Ikeda et al., 2007; Nieto et al., 1996; Viñuelas et al., 2001). For the occurrence of leukocyte migration from blood to the brain, chemokines are key molecules. They compose a superfamily of low molecular weight proteins ( $8-10 \mathrm{kDa}$ ), which act in the immune response, mainly activation and guidance of leukocyte traffic (chemotaxis) (Bendall, 2005; Mantovani, 1999).

Chemokines are divided in four subfamilies, according to the position of cysteine residues: CXC ( $\alpha$-chemokines), CC ( $\beta$-chemokines), $C$ and CX3C (Mantovani, 1999; Peeters et al., 2006). Among the $\beta$ chemokines there are MCPs (monocyte chemoattractant proteins) -1 and -2 (or CCL-2 and CCL-8, respectively); MIPs (macrophage inflammatory proteins) $-1 \alpha$ and $-1 \beta$ (or CCL-3 and CCL-4, respectively); and RANTES (regulated on activation, normal T cell expressed and secreted, or CCL-5), which are highly chemoattractive to monocytes/macrophages, several lymphocytes subsets, dendritic cells and NK cells (Bendall, 2005; Rabin, 2003).

A major representative of $\alpha$-chemokines is CXCL-10 (interferon gamma-induced protein 10 , or IP-10), which main function is to regulate effector Th1 cell migration to the site of inflammation during adaptive immune response (Bendall, 2005; Murphy, 2003). Further, CX3CL-1 (fractalkine) is the only chemokine belonging to the CX3C subfamily. It acts as a chemoattractant and as an adhesion molecule, since it is present in both soluble and membrane-anchored forms. CX3CL-1 is 
expressed by macrophages, dendritic cells, neurons and activated endothelial cells, and it is chemoattractive principally to $\mathrm{T}$ lymphocytes and NK cells (Bendall, 2005; Maeda et al., 2012). In the brain, neurons express elevated concentrations of CX3CL-1, which acts in microglial modulation by interaction with the receptor CX3CR-1, expressed by microglia (Hanisch and Kettenmann, 2007).

Limited studies focused on the neuropathogenesis of VL. We have previously observed the presence of inflammatory stimuli in the brain of infected dogs, such as glial activation, cytokine overexpression and matrix metalloproteinase enzymes (Melo and Machado, 2011; Melo et al., 2013) that could facilitate the accumulation of inflammatory cells, essentially T lymphocytes (Melo et al., 2009). Therefore, since the evidences of brain inflammation during canine VL are robust but the pathogenesis is unclear, we aimed to evaluate T lymphocyte populations and the gene expression of CCL-2, CCL-3, CCL-4, CCL-5, CCL-8, CXCL-10, CX3CL-1 and the receptor CX3CR-1 in the brain of dogs naturally infected by Leishmania spp., comparing with the chemokine profile expressed in the spleen; and attempting to correlate the expression of the chemokines with the clinical stage and the presence of the parasite in the brain, assessed by qPCR, in situ hybridization and immunohistochemistry.

\section{Materials and methods}

\subsection{Animals}

Twenty dogs were included in this study. Fifteen dogs proceeding from the Zoonosis Control Center in the municipality of Araçatuba, São Paulo State, Brazil, were selected as soon as VL diagnosis was achieved by serology (DPP and ELISA, Bio-Manguinhos/Fiocruz, Manguinhos, RJ, Brazil). The age ranged from 1 to 4 years old, 7 males and 8 females. Five uninfected dogs which death was not related to brain disease (i.e. trauma) were included as control.

\subsection{Sampling}

The dogs were euthanized with the owners' permission according to the recommendations of the current VL control program (São Paulo, 2006), using sodium thiopental and potassium chloride. We collected peripheral blood samples in tubes with and without EDTA, and urine samples by cystocentesis; however, the bladder was empty in three animals. Afterwards, we performed necroscopic examinations to evaluate macroscopic alterations and to collect samples of brain and spleen. The brain, representative of the central nervous system (CNS), was considered our organ of interest, while the spleen, representative of the periphery, was considered the target organ of the infection.

From the brain, we collected one hemisphere and stored in $10 \%$ buffered-formalin. After fixation, coronal sections were made and samples containing cerebral cortex, thalamus, hippocampus, pons-medulla oblongata, cerebellum, the ventricular choroid plexi and periventricular white matter were paraffin-embedded, sectioned $(5 \mu \mathrm{m})$ and submitted to hematoxylin and eosin (HE) staining, in situ hybridization and immunohistochemistry. From the other hemisphere, unfixed, we collected a pool of fragments of $0.5 \mathrm{~cm}^{3}$ from the thalamus, hippocampus, piriform/temporal cortex and periventricular white matter, stored in RNAlater (AM7020, Applied Biosystems, Foster City, CA, USA) for RNA extraction, or directly frozen at $-80^{\circ} \mathrm{C}$ for DNA extraction. Regarding the spleen, we performed tissue smears, and we collected fragments in formalin, in RNAlater and to be directly frozen at $-80{ }^{\circ} \mathrm{C}$.

\subsection{Clinical staging}

We performed the complete bloodwork of the animals, using routine methods to determine the serum concentrations of total protein, albumin, urea and creatinine. We determined the serum concentrations of anti-Leishmania antibodies using indirect ELISA (Lima et al., 2005).
Urinalysis and urinary protein/creatinine ratio (UPC; $\{$ urinary protein $\}$ ureatinine $\}$ were also performed. The clinical staging was defined according to Solano-Gallego et al. (2011).

\subsection{In situ hybridization (ISH) to detect Leishmania}

We performed in situ hybridization in brain and spleen sections following Dinhopl et al. (2011). Briefly, slides containing tissue sections were dewaxed in xylene and hydrated in ethanol at $100 \%, 70 \%$, and $50 \%$ and distilled water, followed by incubation with Proteinase $\mathrm{K}$ (S3004, Dako, Carpinteria, CA, USA) during $10 \mathrm{~min}$ and subsequent washing in distilled water, in ethanol $96 \%$ and in isopropanol for 5 min each. The slides were air-dried and frame seals (SLF-1201, BioRad, Hercules, CA, USA) were attached. Then, we added $125 \mu \mathrm{L}$ of hybridization mix containing $15 \mu \mathrm{L}$ of distilled water; $25 \mu \mathrm{L}$ of $20 \times$ SSC buffer; $62.5 \mu \mathrm{L}$ of formamide $50 \% ; 12.5 \mu \mathrm{L}$ of dextran sulfate $50 \%$; 2,5 $\mu \mathrm{L}$ of Denhardt's solution (D2532, Sigma-Aldrich, Saint Louis, MO, USA); $6.25 \mu \mathrm{L}$ of herring sperm DNA (D7290, Sigma-Aldrich); and $1.25 \mu \mathrm{L}$ of $3^{\prime}$-digoxigenin-conjugated probe (Eurofins MWG Operon, Huntsville, AL, USA). The probe, 5'-ACGGGGATGACACAATAGAGCTTC TCC-3', in final concentration of $100 \mathrm{ng} / \mathrm{mL}$, detects a segment of the $5.8 \mathrm{~S}$ ribosomal RNA of Leishmania genus. The slides were incubated at $95{ }^{\circ} \mathrm{C}$ for $6 \mathrm{~min}$, immediately cooled in ice, and then incubated at 40 ${ }^{\circ} \mathrm{C}$ for $14-16 \mathrm{~h}$ in humid chamber. Afterwards, the slides were washed in $2 \times$ SSC, $1 \times$ SSC and $0.1 \times$ SSC buffer for 5 min each, followed by incubation with the anti-digoxigenin antibody conjugated to alkaline phosphatase, diluted in TBS $(1: 200 ; 11093274910$, Roche Diagnostics, Indianapolis, IN, USA) for $1 \mathrm{~h}$ and then washed in TBS. Visualization was achieved using NBT/BCIP (nitro blue tetrazolium/5-bromo-4chloro-3-indolyl-phosphate) (11681451001, Roche Diagnostics) for $1 \mathrm{~h}$ in the dark. The reaction was stopped with TE buffer ( $\mathrm{pH} 8.0$ ) for $10 \mathrm{~min}$ followed by washing in distilled water. The slides were counterstained with Mayer's hematoxylin and mounted in aqueous medium (Faramount, S3025, Dako). Parasites were identified by a dark purple signal (Supplemental Fig. 1).

\subsection{Leishmania DNA quantification}

We extracted total DNA from tissue fragments (spleen and a pool of brain fragments) weighting ca. $25 \mathrm{mg}$ using the DNeasy blood \& tissue kit (69506, Qiagen, Hilden, Germany) according to the manufacturer's protocol. The DNA was quantified with a NanoDrop spectrophotometer (260/280 ratio between 1.8 and 2.0). We performed qPCRs using CFX96 ${ }^{\mathrm{TM}}$ Real-time System (Bio-Rad), SYBR Green PCR Master Mix (4309155, Applied Biosystems) and $900 \mathrm{nM}$ of each primer (sense: 5'CCTATTTTACACCAACCCCCAGT-3'; anti-sense: 5'-GGGTAGGGGCGTTC TGCGAAA- $3^{\prime}$ ) which amplify a 116 bp fragment of the minicircle kinetoplast DNA (kDNA) of Leishmania spp. (Ranasinghe et al., 2008), in a total volume of $25 \mu \mathrm{L}$. The amplification conditions were the following: $94{ }^{\circ} \mathrm{C}$ for $2 \mathrm{~min}$ and 40 cycles of $94^{\circ} \mathrm{C}$ for $15 \mathrm{~s}$ and $60^{\circ} \mathrm{C}$ for $1 \mathrm{~min}$. Then, the samples were submitted to a melt curve from $60{ }^{\circ} \mathrm{C}$ to $95^{\circ} \mathrm{C}$; with a $0.5^{\circ} \mathrm{C}$ increase every $5 \mathrm{~s}$. We assessed the absolute quantification using a standard curve containing serial dilutions (from $10^{-1}$ to $10^{6}$ promastigotes) of L. infantum DNA (MHOM/BR/72/LD46). The lower limit of positivity (cut-off value) was established using the results obtained from spleens and brains of uninfected dogs.

\subsection{Chemokine gene expression}

We extract the RNA from brain and spleen samples stored in RNAlater using the RNeasy Mini kit (74104, Qiagen) following the manufacturer's instructions. Total RNA was quantified in a NanoDrop spectrophotometer (260/280 ratio between 2.0 and 2.3) and then submitted to genomic DNA elimination and reverse transcription using 
the QuantiTect Reverse Transcription kit (205314, Qiagen) following the manufacturer's instructions.

We performed qPCRs using CFX96 ${ }^{\mathrm{TM}}$ Real-Time System (Bio-Rad), TaqMan master mix (4304437, Applied Biosystems), and customized gene expression assays (4351372 and 4331182, Applied Biosystems): CCL-2 (Cf02671955_g1), CCL-3 (Cf02671956_m1), CCL-4 (Cf02622476_m1), CCL-5 (Cf02622325_m1), CCL-8 (Cf02622478_m1), CXCL10 (Cf02622528_m1), CX3CL1 (Cf02651336_m1) and CX3CR1 (Cf02695529_s1). The reference genes were RPL32 (ribosomal protein L32; Cf03986518_m1) and G3PDH (glyceraldehyde 3-phosphate dehydrogenase; Peeters et al., 2006). The amplification conditions were as follows: $55^{\circ} \mathrm{C}$ for $2 \mathrm{~min}, 95^{\circ} \mathrm{C}$ for $10 \mathrm{~min}, 45$ cycles of $95{ }^{\circ} \mathrm{C}$ for $15 \mathrm{~s}$ and $60{ }^{\circ} \mathrm{C}$ for $1 \mathrm{~min}$. For each target, we obtained values of reaction efficiency from amplification of seven serial dilutions of a pool of cDNA). The relative gene expression was achieved using the REST ${ }^{\circledR}$ software (Pfaffl et al., 2002) which indicates how many times (fold change) the expression of a target gene is higher (up-regulated) or lower (down-regulated) in the infected group when compared with the control group.

\subsection{T lymphocyte characterization and Leishmania detection in the brain by immunohistochemistry}

To detect Leishmania parasites, IgG and CD3 + T cells in the brain, after dewaxing the tissue sections, endogenous peroxidase activity was blocked by incubating sections in $2 \%(\mathrm{v} / \mathrm{v})$ hydrogen peroxide 30 vol. diluted in 50\% (v/v) methanol for $30 \mathrm{~min}$. Pre-treatments for antigen retrieval were done according to the primary antibody specification (Table 1). Non-specific binding was blocked with $3 \%(\mathrm{w} / \mathrm{v})$ nonfat dry milk in PBS (phosphate-buffered saline) pH 7.2 for 30 min. Sections were incubated with the primary antibody (Table 1) for $18-22 \mathrm{~h}$ at $4{ }^{\circ} \mathrm{C}$ in a humidified chamber. Slides were washed in PBS, incubated with a biotinylated secondary antibody and with streptavidin-HRP complex (LSAB + Kit, Dako, K0690) according to the manufacturer's instructions. The reaction was developed with 3,3'-diaminobenzidine (Dako, K3468). Regarding the detection of CD4+ cells and CD8 + cells; we used Novocastra antibodies (Leica, Newcastle upon Tyne, United Kingdom), which are able to detect these molecules in paraffin sections (Table 1), and the Catalyzed Signal Amplification System (CSA; Dako, K1500), following the manufacturer's specification. The slides were then counterstained with Harris's hematoxylin, dehydrated, cleared, and mounted with coverslips. Tissue samples were examined by light microscopy and we measured the positivestained area using a computerized image-analysis software (ImagePro Plus 6.1, Media Cybernetics) as per Melo and Machado (2011). The intensity of the positive staining was evaluated in a total area of $814.554 .4 \mu \mathrm{m}^{2}$. The results are expressed as the percentage of the tissue's positive-stained area. All analyses were done "blindly", without knowledge of the experimental groups. Human tonsil and canine lymph node sections were used as positive controls (Supplemental Figs. 2-3).

\subsection{Statistical analyses}

Correlation between chemokines was assessed by the Spearman test. Differences for T lymphocyte subpopulations were determined using the Mann-Whitney test. Values of $\mathrm{P}<0.05$ were considered statistically significant. Data were expressed as the median and the interquartile range (IQR). All statistical analyses were performed using Prism software (v6.05, GraphPad, La Jolla, CA, USA).

\subsection{Ethical issue}

All procedures were performed according to the Brazilian College of Animal Experimentation, and approved by the Institutional Ethics Committee (CEUA, FMVA-UNESP, process \#2012-01093).

\section{Results}

\subsection{Clinical staging}

The most common clinical sign was skin disease (53.3\%, 8/15), including alopecia, nasal hyperkeratosis, ulcers and seborrhea, splenomegaly $(46.7 \%, 7 / 15)$, followed by lymphadenopathy $(40 \%, 6 / 15)$, conjunctivitis $(40 \%, 6 / 15)$, and ear pinna necrosis $(40 \%, 6 / 15)$. Onycogryphosis and cachexia were observed in $26.7 \%$ (4/15) of the dogs.

Regarding laboratorial findings, 93.3\% (14/15) of the infected dogs presented normocytic-normochromic anemia, 53.3\% (8/15) presented lymphocytopenia and 66.7\% (10/15) presented thrombocytopenia. All infected dogs presented hypoalbuminemia and reduced albumin/ globulin ratio. Azotemia was evident in $26.7 \%$ (4/15) of the infected dogs, and $66.7 \%$ ( 8 out of 12 urine samples) presented increased UPC. All infected dogs presented positive concentrations of serum anti-Leishmania antibodies, 6.7\% (1/15) with low intensity (from cutoff value 0.27 up to 0.4$), 67.7 \%$ (10/15) with medium intensity (0.4 up to 0.81 ), and $26.7 \%$ (4/15) with high intensity (over 0.81 ).

Direct parasitological examinations in spleen smears were positive in 33.3\% (5/15) of the infected dogs; however, all the spleens of infected dogs were positive in qPCR. Therefore, we classified 20\% (3/15) of the dogs in stage I, 53.3\% (8/15) in stage II, 20\% (3/15) in stage III and 6.7\% $(1 / 15)$ in stage IV of the disease. Individual values of all measured data are available in Supplemental Tables 1-4.

\subsection{Brain histopathology}

The most common histopathological findings in the brain of the infected dogs with VL were inflammatory changes (Fig. 1), ranging from discrete to severe, including leptomeningitis $(80.0 \%, 12 / 15)$, choroiditis (73.3\%, 11/15), subventricular gliosis $(73.3 \%, 11 / 15)$ and parenchymal mononuclear perivascular cuffs $(53.3 \%, 8 / 15)$. Further, we also observed vascular congestion, microhemorrhages, satellitosis/neuronophagia, and glial nodules. No association between clinical signs and brain histopathological alterations was observed. We did not detect in the parasite in the brain sections stained with HE, however, the parasites were clearly observed in spleen sections.

\subsection{Localization of Leishmania parasites in the brain}

We did not detect Leishmania parasites within the brain of infected dogs using neither in situ hybridization nor immunohistochemistry. Nevertheless, by means of ISH, we observed a perivascular positive staining in the choroid plexus of $78.6 \%$ of the dogs (11 out of 14 dogs

Table 1

Panel of antibodies used in the immunohistochemical analyses in the brain of dogs with visceral leishmaniasis.

\begin{tabular}{|c|c|c|c|c|}
\hline Antibody & Specificity & Dilution & Pre-treatment & Source \\
\hline Polyclonal rabbit anti-CD3 & T cells & $1: 200$ & Citrate $10 \mathrm{mM} \mathrm{pH} 6.0$ in steamer for $30 \mathrm{~min}$ & Dako, A0452 \\
\hline Monoclonal mouse anti-CD4 clone 1F6 & CD4 + cells & $1: 100$ & EDTA $1 \mathrm{mM}$ pH 8,0 in steamer for $30 \mathrm{~min}$ & Novocastra, NCL-L-CD4-1F6 \\
\hline Monoclonal mouse anti-CD8 clone 1A5 & CD8 + cells & $1: 100$ & Citrate $10 \mathrm{mM} \mathrm{pH} 6.0$ in steamer for $30 \mathrm{~min}$ & Novocastra, NCL-CD8-295 \\
\hline Polyclonal rabbit anti-dog IgG & $\operatorname{IgG}$ & $1: 100$ & Citrate $10 \mathrm{mM} \mathrm{pH} 6.0$ in steamer for $30 \mathrm{~min}$ & Sigma-Aldrich, A6792 \\
\hline Heterologous hyperimmune canine serum ${ }^{\mathrm{a}}$ & Leishmania sp. & $1: 100$ & Citrate $10 \mathrm{mM} \mathrm{pH} 6.0$ in steamer for $30 \mathrm{~min}$ & - \\
\hline
\end{tabular}

a According to Tafuri et al. (2004). 


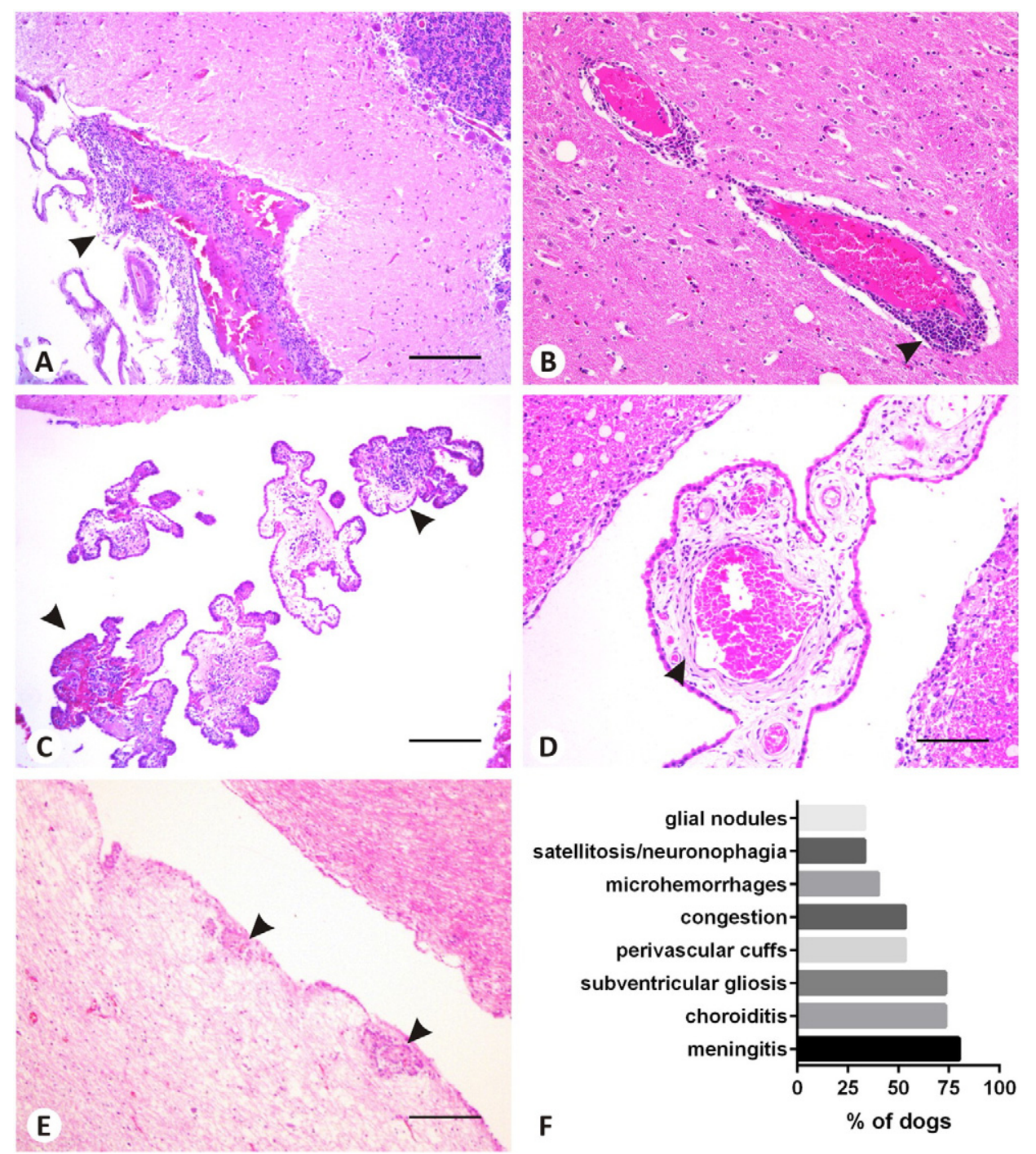

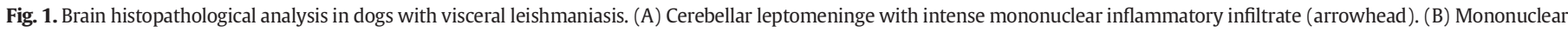

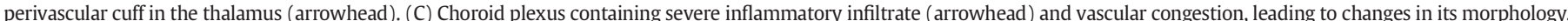

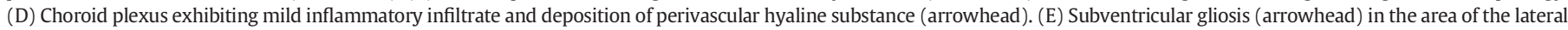

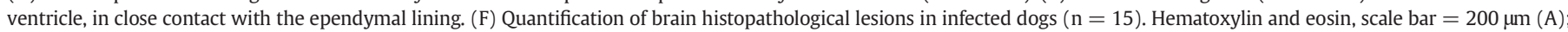
$100 \mu \mathrm{m}$ (B, C, E); $50 \mu \mathrm{m}$ (D).

where this structure was present in the sections) (Fig. 2A-C). In order to better describe this finding, we performed paired HE and anti-IgG immunohistochemical analyses in the same sections containing positive ISH staining in the choroid plexus. In HE-stained tissues, there was no detectable parasite or parasitized cell, but inflammatory infiltrate and stroma thickening (Fig. 2B). Immunohistochemistry revealed intense IgG deposition in the choroid plexus' stroma (Fig. 2D). Therefore, we classified this alteration as perivascular Leishmania DNA/IgG deposition.

\subsection{Leishmania DNA quantification in the brain}

We were able to detect and quantify the parasite DNA in the brain of the infected dogs using qPCR. With large variability, the brain presented parasite loads ranging from $1.1 \times 10^{1}$ to $6.6 \times 10^{3}$ parasites $/ 25 \mathrm{mg}$ of tissue (Fig. 3). Nevertheless, only $53.3 \%$ (8/15) of the dogs presented values higher than the lower limit of positivity $\left(3.9 \times 10^{1}\right)$. There was no correlation regarding brain parasite load and the clinical stages. On the other hand, all the spleens of infected dogs were positives, ranging from $1.5 \times 10^{2}$ to $7.2 \times 10^{5}$ parasites $/ 25 \mathrm{mg}$ of tissue (cut-off value of $\left.1.3 \times 10^{0}\right)$. Comparing brain and spleen, we noticed a positive correlation between parasite load in these organs $(r=0.610 ; \mathrm{P}=0.018)$.

\subsection{Chemokine gene expression in the brain and in the spleen of infected dogs}

In order to evaluate the up- or down-regulation of selected chemokine gene expression in the brain and spleen of dogs with VL, we used the REST ${ }^{\circledR}$ method, which expresses how many times (fold changes) the target gene is more or less expressed in the infected dogs, compared with the control ones. Reaction efficiency values, determination coefficients and angular coefficients of each gene are shown in Table 2.

All chemokines were up-regulated in the spleen of infected dogs (Table 2; 4). No changes were observed for the receptor CX3CR-1. No correlation between the clinical stage and parasite load with chemokine expression was noticed, however, in the spleen; CCL-3, CCL-4, CCL-5 and CCL- 8 were positively correlated among them, as well as CCL-2 with CCL-8 and CXCL-10.

In the brain of infected dogs, only the gene expression of CCL-3 (19.9-fold), CCL-4 (16.2-fold) and CCL-5 (20.3-fold) was significantly 

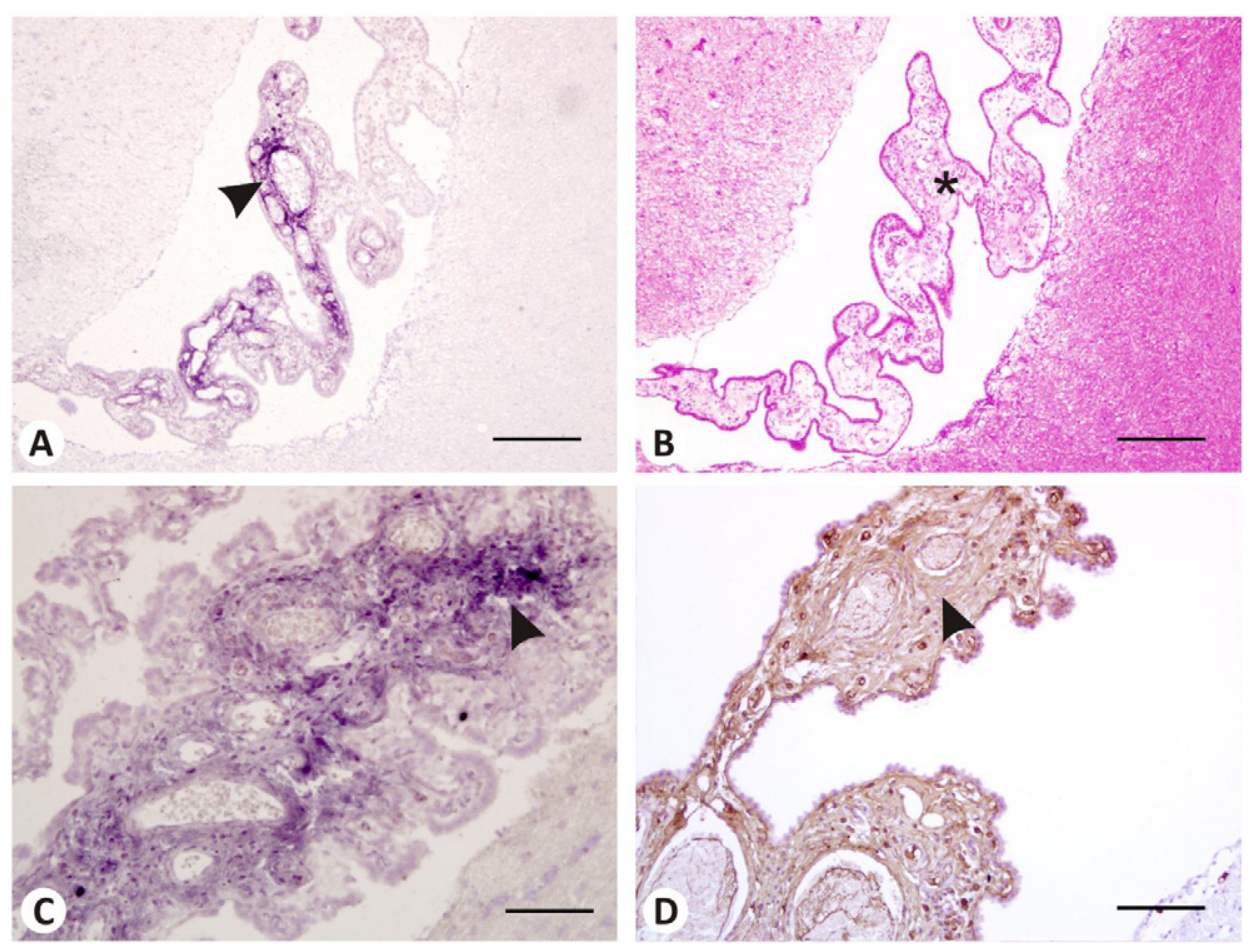

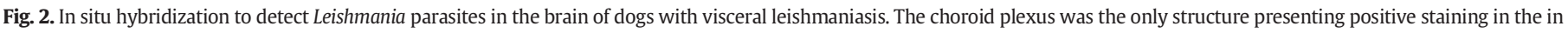

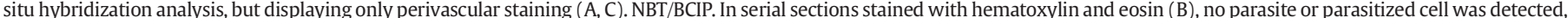

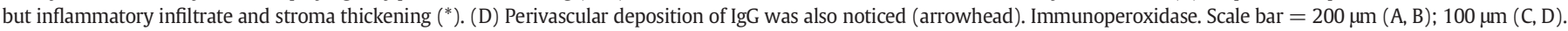

up-regulated (Table 2; Fig. 4), with strong positive correlation among them (Fig. 5A-C). No correlation between the chemokines in the brain and clinical stage or parasite load was observed. As expected in a natural infection sampling, we observed important individual variability in all targets, nevertheless, different from the other targets, the distribution

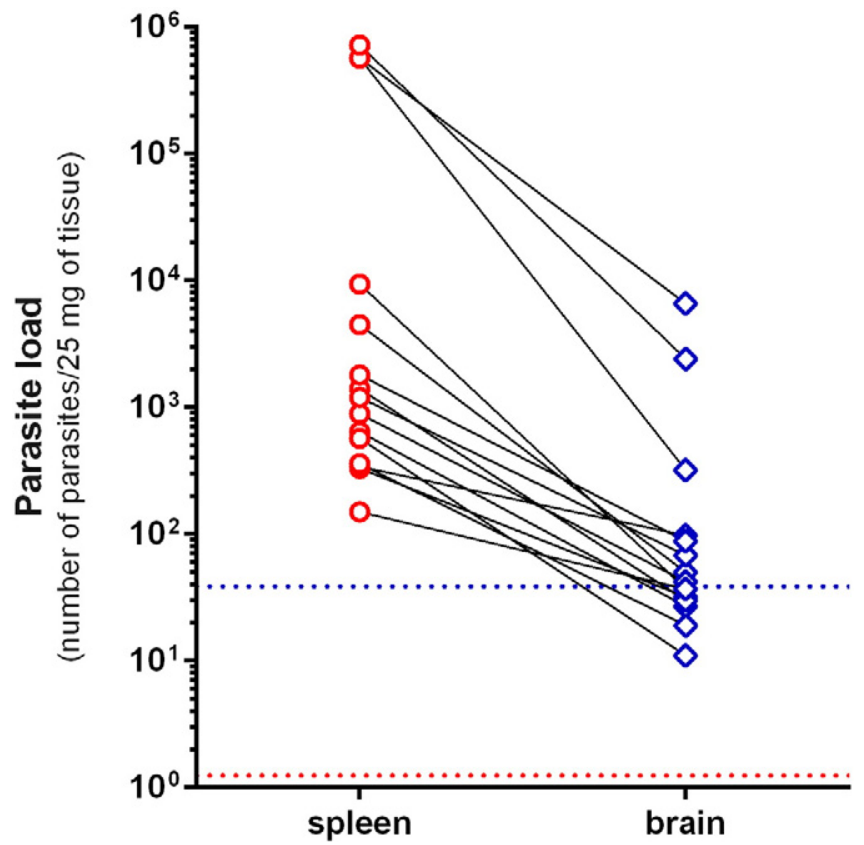

Fig. 3. Individual parasite load determination in the spleen and in the brain of dogs with visceral leishmaniasis. Black lines connect the spleen and the brain values of the same dog. The dotted lines represent the lower limit of positivity: spleen $=$ red dotted line $\left(1.3 \times 10^{0}\right.$ parasites $)$, brain $=$ blue dotted line $\left(3.9 \times 10^{1}\right.$ parasites $)$. of CXCL-10 gene expression displayed a specific pattern in the infected dogs, with 10 dogs with expression values similar to the control dogs, and a small subpopulation ( $\mathrm{n}=5$; \#GD3, \#GD4, \#GD5, \#GD9, \#GD10) with evident higher gene expression. When evaluated separately, this subpopulation presented 83.8 -fold more CXCL-10 expression than the controls (Fig. $5 \mathrm{D} ; \mathrm{P}=0.002$ ). Even in this particular case, we detected no correlation to the clinical stages, parasite load, and inflammatory status or to other chemokines. CXCL-10 was correlated to CCL-2 in the spleen, and besides the absence of changes in the gene expression of CCL-2 in the brain, we observed a trend to positive correlation between CXCL-10 and CCL-2 in the brain (Fig. 5E).

\subsection{T lymphocytes in the brain}

$\mathrm{CD} 3+$ cells were the main component of the inflammatory infiltrate in the brain of the infected dogs, located in the leptomeninges, subependymal area, choroid plexus and in the parenchymal perivascular cuffs (Fig. 6A-C). Infected dogs presented a median value of $0.57 \%$ (IQR 0.73 ) whereas control dogs presented solely $0.04 \%$ (IQR

Table 2

Reaction efficiency values (E), determination coefficients $\left(r^{2}\right)$ and angular coefficients (slope) of the RT-qPCRs of each evaluated gene, and P-values regarding the relative gene expression of the target genes in the spleen and in the brain.

\begin{tabular}{lrrlll}
\hline \multirow{2}{*}{ Target } & $\mathrm{E}(\%)$ & \multicolumn{2}{l}{$\mathrm{r}^{2}$} & Slope & \multicolumn{2}{l}{ P-value } \\
\cline { 5 - 6 } & & & & Spleen & Brain \\
\hline G3PDH & 106.6 & 0977 & -3173 & - & - \\
RPL-32 & 100.2 & 0.994 & -3.318 & - & - \\
CCL-2 & 101.8 & 0.998 & -3.279 & $0.000 \uparrow$ & 0.337 \\
CCL-3 & 99.1 & 0.999 & -3.344 & $0.000 \uparrow$ & $0.010 \uparrow$ \\
CCL-4 & 96.4 & 0.999 & -3.412 & $0.002 \uparrow$ & $0.007 \uparrow$ \\
CCL-5 & 101.7 & 0.992 & -3.282 & $0.000 \uparrow$ & $0.018 \uparrow$ \\
CCL-8 & 99.6 & 0.994 & -3.330 & $0.000 \uparrow$ & 0.353 \\
CXCL-10 & 101.0 & 0.993 & -3.299 & $0.000 \uparrow$ & 0.388 \\
CX3CL-1 & 99.4 & 0.995 & -3.337 & $0.005 \uparrow$ & 0.431 \\
CX3CR-1 & 104.6 & 0.994 & -3.217 & 0.168 & 0.700 \\
\hline
\end{tabular}

$\uparrow$ indicates significative up-regulation. 


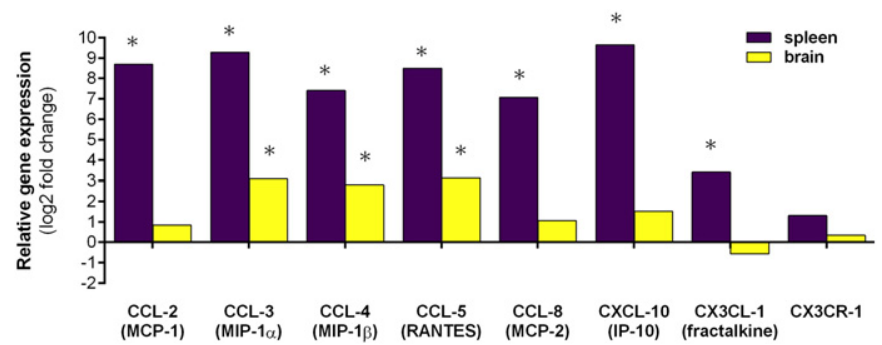

Fig. 4. Relative gene expression of chemokines in the spleen and in the brain of dogs with visceral leishmaniasis. The values are expressed as fold changes $(\log 2)$. Positive values indicate up-regulation and negative values indicate down-regulation, when compared with the control dogs. The normalization factor was the reference genes G3PDH and RPL-32. * indicates $\mathrm{P}<0.05$.

$0.02)$ of immunostained area $(P=0.0002)$. $C D 4$ and $C D 8$ were rarely detected, only in the infected dogs (Fig. 6D).

\section{Discussion}

We observed the presence of inflammation, chemokine expression and parasite DNA in the brain of dogs with VL. The sample population included dogs belonging to the four clinical stages of the classification proposed by Solano-Gallego et al. (2011). We chose the spleen as the control organ of the infection since it is considered one of the parasite's targets and it is the focus of inflammation during the disease. The cytohistopathological findings in this organ support this statement, as they were coherent with the classical lesions previously reported (Lima et al., 2012). Regarding the brain, we observed important inflammatory alterations, with different intensities, ranging from discrete to intense, corroborating previous studies from our research group and others (Márquez et al., 2013; Melo et al., 2013; Viñuelas et al., 2001); consequently, we can confirm that the brain is also affected during VL, even in the absence of neurological symptoms. Nevertheless, its pathogenesis is still unclear.
One possible stimulus to trigger the inflammatory response in the brain would be the presence of the parasite itself. Infection by other members of the Trypanosomatidae family leads to parasite accumulation in the brain. Trypanosoma brucei invades the CNS via the choroid plexus and the circumventricular organs (Masocha et al., 2007). Trypanosoma evansi was present in the brain of naturally-infected horses (Rodrigues et al., 2009) as well as Trypanosoma cruzi in immunodeficient humans (Javier et al., 1998). Leishmania on the other hand has been sporadically noticed in the CNS, using either histology or immunohistochemistry (Márquez et al., 2013; Nieto et al., 1996; Viñuelas et al., 2001).

Even though in situ hybridization reactions were effective to detect the parasite in the spleen, no parasite was observed in the brain. Nevertheless, we noticed perivascular staining exclusively at the choroid plexus, consistent with perivascular parasite DNA deposition. In oncology, a similar finding is named Azzopardi phenomenon, which corresponds to DNA deposition around blood vessels in areas of tissue necrosis, since massive cell lysis releases large amounts of nucleic acids (Pritt and Cooper, 2003). To our knowledge, there is no description of Azzopardi phenomenon in infectious diseases; however, there are reports of free DNA fragments, smaller than $300 \mathrm{bp}$, circulating in biological fluids such as plasma, serum and urine. These fragments are called cell-free DNA, and they could be originated from the host cells' death (selfDNA) or from microorganisms' life cycle (replication, maturation and death) (Dwivedi et al., 2012; Green et al., 2009), including Leishmania spp. (Franceschi et al., 2007).

At histological examination, we did not detect parasites in the choroid plexus in the areas where in situ hybridization revealed perivascular Leishmania DNA deposition. The presence of parasite DNA in the brain was corroborated by qPCR, and even though positive, the average parasite load in the brain was low. Furthermore, the perivascular deposits in the choroid plexus were hyaline, consistent with IgG deposition, and not basophilic as expected for nucleic acids. Nevertheless, DNA-containing immune complexes or even small amounts of cell-free DNA (Atkins et al., 1972; Falangola et al., 1995)
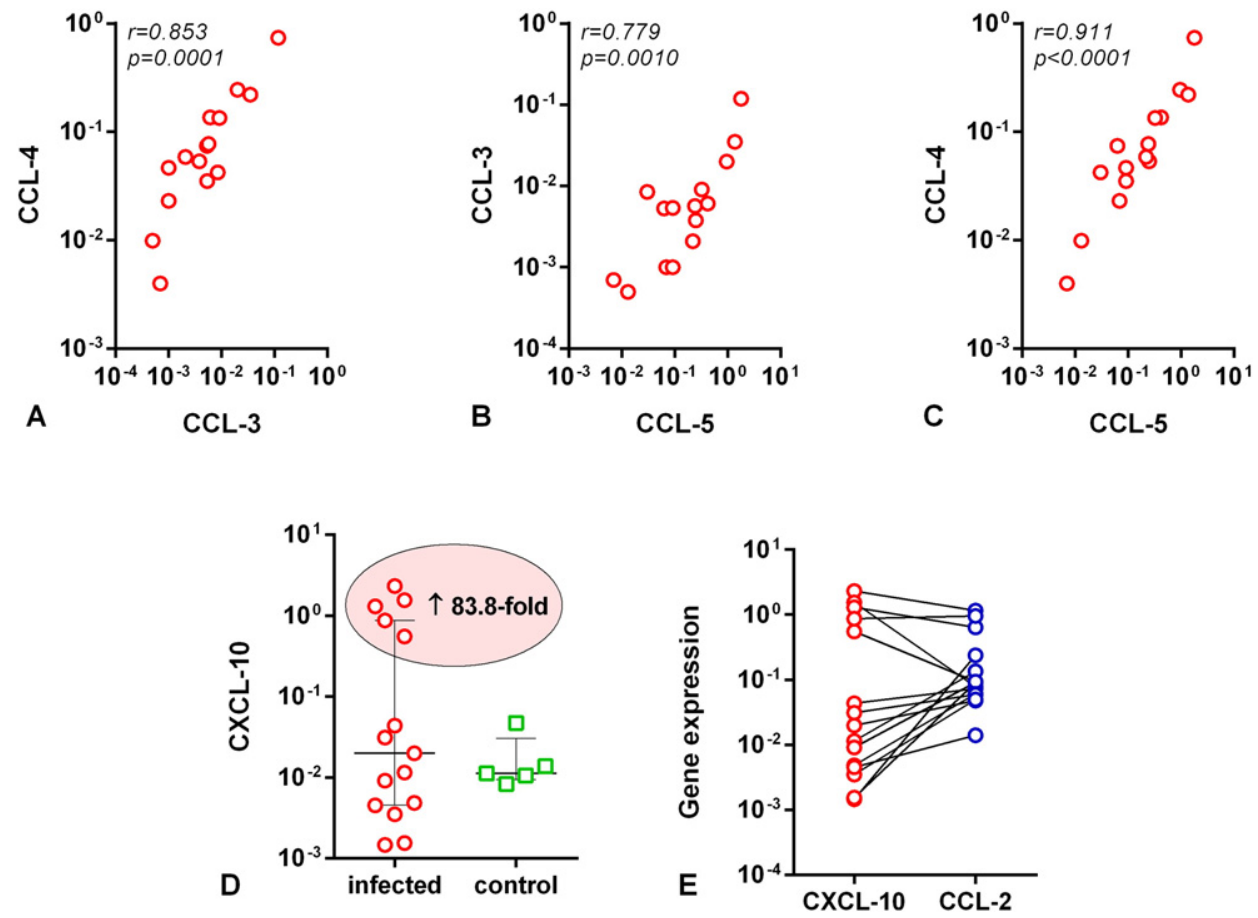

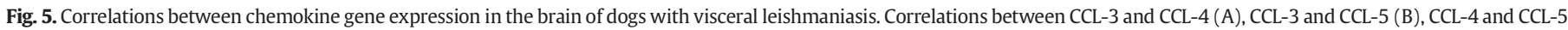

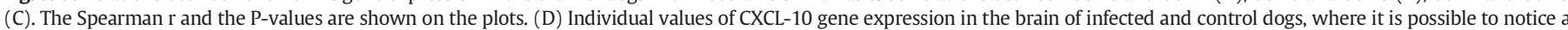

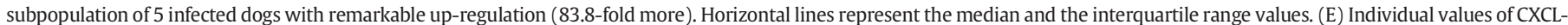

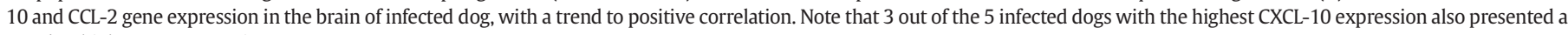
trend to high CCL-2 expression. 

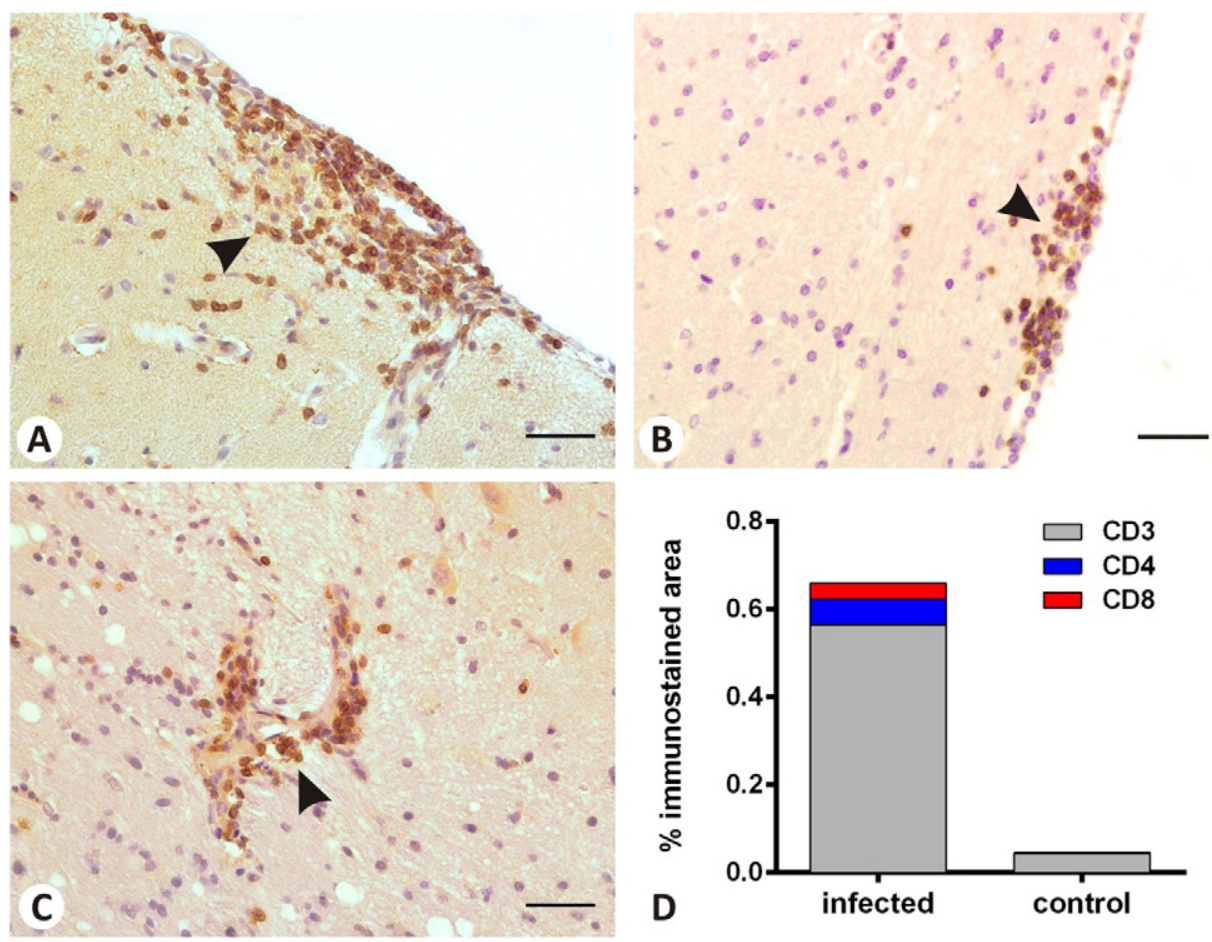

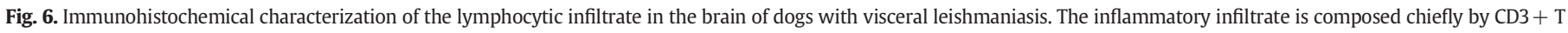

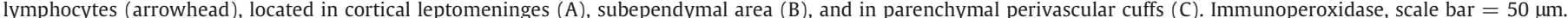

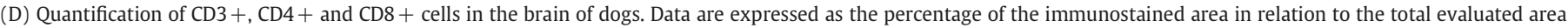
$\left(814.554 .4 \mu \mathrm{m}^{2}\right)$. Columns represent the median value of the groups.

may arrive to the choroid plexus stroma by vascular fenestrations and inflammatory endothelial lesions (Wolburg and Paulus, 2010), which could be enough to render positive a test based on DNA detection and to initiate inflammation. High concentrations of circulating immune complexes have already been detected in VL, which has been correlated to glomerulonephritis and necrotizing vasculitis (Alvar et al., 2004; Brandonisio et al., 1990). The parasite DNA could also trigger inflammation by activation of Toll-like receptors, probably TLR-9, which binds DNA and that have already been detected in the choroid plexus of dogs with VL (Melo et al., 2014).

The first report of Leishmania detection in the choroid plexus in dogs was published by Nieto et al. (1996). Thereafter, histological alterations were described in the choroid plexus of dogs with VL, but with no detectable parasites (Pumarola et al., 1991; Ikeda et al., 2007; Melo and Machado, 2009). Since we have been noticing alterations in the choroid plexus and brain of dogs with VL with no detectable parasites within the CNS (Melo and Machado, 2009, 2011; Melo et al., 2009, $2013,2014)$, we can propose two hypotheses to brain inflammation during VL: (1) parasite-dependent, where the parasites enter the CNS, stimulate inflammation and may be latter destroyed, rendering difficult their detection, and (2) parasite-independent, where peripheral stimuli (parasite antigens/DNA, inflammatory mediators) reach the CNS and trigger inflammation. In one way or another, brain inflammation occurs in dogs with VL.

The study of the immune response in the CNS during VL is infrequent and the evaluation of the chemokine profile in infected dogs is rare. Glial cells and even leukocytes within the inflammatory infiltrates may produce chemokines. We detected a specific pattern of chemokine expression, with up-regulation of CCL-3, CCL-4 and CCL-5 in the brain of infected dogs. CCL-5 is a well-characterized chemokine related to the recruiting and guidance of $\mathrm{T}$ lymphocytes towards the focus of inflammation. CCL3 and CCL-4, despite being initially related to macrophages and NK cell activation, they present a selective chemoattraction to CD8 and CD4 activated T lymphocytes, respectively (Taub et al., 1993). Moreover, CCL-4 and
CCL-5 promoted CD4 T lymphocyte adhesion to the endothelium activated by IFN- $\gamma$ and TNF- $\alpha$ (Quandt and Dorovini-Zis, 2004).

The interaction of these three chemokines, CCL-3, CCL- 4 and CCL-5, is coherent with the predominance of T lymphocytes previously described in the brain of dogs with VL (Melo et al., 2009). In accordance, resistant mice presented low expression of CCL-3, CCL-4 and CCL-5, resulting in absence of $\mathrm{CD} 8 \mathrm{~T}$ lymphocytes in the inflammatory infiltrate in a murine model of cerebral malaria (Clark and Phillips, 2011). The overexpression of CCL- 5 by a recombinant rabies virus promoted severe blood-brain barrier disruption associated with chemokine production and inflammatory cell infiltration (Zhao et al., 2009). Despite immunohistochemistry being not the ideal technique to characterize lymphocyte subsets, we have shown here that the main inflammatory cells in the brain are $\mathrm{CD} 3+\mathrm{T}$ lymphocytes, and along with a minimal detection of $\mathrm{CD} 4$ or $\mathrm{CD} 8$, these cells might belong to the $\mathrm{CD} 3+/ \mathrm{CD} 4-/ \mathrm{CD} 8-$ cell subset, nevertheless, an extensive study of the T cell subpopulations in the brain of dogs with VL using flow cytometry is required. Recently, different subsets of T lymphocytes gained focus on parasitic diseases, especially the double negative (DN) T cells, which express neither CD4 nor CD8, including $\alpha \beta$ DN T cells, $\gamma \delta$ DN T cells, NK T cells (D'Acquisto and Crompton, 2011). In cases of human cutaneous leishmaniasis, elevated numbers of circulating $\alpha \beta$ DN T cells are described, associated with elevated production of IFN- $\gamma$ and TNF- $\alpha$ and high levels of the activation markers CD69 and CD56 (Antonelli et al., 2006; Gollob et al., 2008). Further, the association between NK T cells and immune response during VL has already been studied in the liver of mice infected with $L$. donovani (Amprey et al., 2004).

Regarding other protozoan diseases in the brain, NK T cells have been related to IFN- $\gamma$ production and recruitment of T lymphocytes via CXCL-10/CXCR-3 during cerebral malaria (Hansen et al., 2003; Hunt et al., 2014). Additionally, in murine toxoplasmic encephalitis, up-regulation of CCL- 5 dependent on IFN- $\gamma$ was described as protective, but when accompanied by up-regulation of CXCL-10 (Wen et al., 2010). IFN- $\gamma$-dependent CXCL10 was also essential to accumulation of T cells 
and parasites in the brain during experimental African trypanosomiasis (Amin et al., 2009).

CXCL-10 presented an interesting pattern of expression in the infected dogs. Whereas $67.7 \%$ of the infected dogs presented no alterations for this chemokine, 33.3\% (5/15) of them presented a remarkable overexpression of CXCL-10 in the brain, with no correlation with clinical stages, parasite load or brain inflammatory infiltrate. The occurrence of upregulation of CXCL-10, even in few dogs, is supported by upregulation of IFN- $\gamma$, previously reported in the brain of dogs with VL (Melo et al., 2013). The high variation of CXCL-10 expression as well as the low average CCL-2 expression in the brain could be related to other individual factor not evaluated in this study, such as specific $\mathrm{T}$ cell subsets or time of infection, similar to what occurs in the spleen of experimentally infected dogs (Strauss-Ayali et al., 2007).

CCL-8, CX3CL-1 and CX3CR-1 presented no alterations in the infected dogs; therefore, these chemokines apparently are not involved in the brain inflammation during VL. In the spleen, on the other hand, all chemokines presented up-regulation in the dogs with VL. Similarly, the spleens of naturally and experimentally infected dogs also presented overexpression of CCL-2, CCL-3, CCL-5 e CXCL-10 (Strauss-Ayali et al., 2007). Nascimento et al. (2013) detected up-regulation only for CXCL10 in the spleen of naturally-infected dogs, however, when comparing asymptomatic and symptomatic dogs, CCL-2, CCL- 5 and CXCL-10 were up-regulated and CCL-4 was down-regulated. The liver of dogs with VL presented generalized decrease of chemokine expression (Nascimento et al., 2013). CCL-2, CCL-4 and CCL-5 were overexpressed in the skin of dogs with $\mathrm{VL}$, with positive correlation with the parasite load (Menezes-Souza et al., 2011). In our study, none of the variables correlated with the clinical stage, however, all the dogs were symptomatic and we used a classification based on a four-point scale (SolanoGallego et al., 2011), which could have dispersed the data, differently from the dichotomous classification asymptomatic-symptomatic.

This variation of chemokine patterns according to the organ corroborates not only the idea of immune compartmentalization during VL (Reis et al., 2009), providing a favorable environment to the accumulation of different cell populations, but also that the brain is a proinflammatory environment, propitious to a selective $\mathrm{T}$ lymphocyte accumulation, even in the absence of the parasite. Indeed, not the parasite itself, but rather its DNA seems to act as a trigger to brain inflammation, together with chronic systemic inflammation. Therefore, our data highlights the need to focus on brain involvement during VL, which has been neglected during the peripheral infection by Leishmania parasites.

\section{Acknowledgments}

This study was supported by Fundação de Amparo à Pesquisa do Estado de São Paulo (FAPESP) [grant number 2012/23950-1]. G.D. Melo was supported by a FAPESP doctoral fellowship [grant number 2012/10415-0].

\section{Appendix A. Supplementary data}

Supplementary data associated with this article can be found in the online version, at http://dx.doi.org/10.1016/j.jneuroim.2015.10.004. These data include the Google map of the most important areas described in this article.

\section{References}

Alvar, J., Cañavate, C., Molina, R., Moreno, J., Nieto, J., 2004. Canine leishmaniasis. Adv. Parasitol. 57, 1-88.

Amin, D.N., Rottenberg, M.E., Thomsen, A.R., Mumba, D., Fenger, C., Kristensson, K., Büscher, P., Finsen, B., Masocha, W., 2009. Expression and role of CXCL10 during the encephalitic stage of experimental and clinical African trypanosomiasis. J. Infect. Dis. 200, 1556-1565.
Amprey, J.L., Im, J.S., Turco, S.J., Murray, H.W., Illarionov, P.A., Besra, G.S., Porcelli, S.A Späth, G.F., 2004. A subset of liver NK T cells is activated during Leishmania donovani infection by CD1d-bound lipophosphoglycan. J. Exp. Med. 200, 895-904.

Antonelli, L.R.V., Dutra, W.O., Oliveira, R.R., Torres, K.C.L., Guimarães, L.H., Bacellar, O. Gollob, K.J., 2006. Disparate immunoregulatory potentials for double-negative $(\mathrm{CD} 4(-) \mathrm{CD} 8(-)) \alpha \beta$ and $\gamma \delta$ T cells from human patients with cutaneous leishmaniasis. Infect. Immun. 74, 6317-6323.

Atkins, C.J., Kondon, J.J.J., Quismorio, F.P., Friou, G.J., 1972. The choroid plexus in systemic lupus erythematosus. Ann. Intern. Med. 76, 65-72.

Baneth, G., Koutinas, A., Solano-Gallego, L., Bourdeau, P., Ferrer, L., 2008. Canine leishmaniosis - new concepts and insights on an expanding zoonosis: part one. Trends Parasitol. 24, 324-330.

Barbiéri, C.L., 2006. Immunology of canine leishmaniasis. Parasite Immunol. 28, 329-377.

Bendall, L., 2005. Chemokines and their receptors in disease. Histol. Histopathol. 20, 907-926.

Brandonisio, O., Carelli, G., Altamura, M., Varvara, B., Ceci, L., 1990. Circulating immune complexes and autoantibodies in canine leishmaniasis. Parassitologia 32, 275-281.

Chappuis, F., Sundar, S., Hailu, A., Ghalib, H., Rijal, S., Peeling, R.W., Alvar, J., Boelaert, M., 2007. Visceral leishmaniasis: what are the needs for diagnosis, treatment and control? Nat. Rev. Microbiol. 5, 873-882.

Clark, C.J., Phillips, R.S., 2011. Cerebral malaria protection in mice by species-specific plasmodium coinfection is associated with reduced CC chemokine levels in the brain. Parasite Immunol. 33, 637-641.

D'Acquisto, F., Crompton, T., 2011. CD3 + CD4 - CD8 - (double negative) T cells: saviours or villains of the immune response? Biochem. Pharmacol. 82, 333-340.

Dinhopl, N., Mostegl, M.M., Richter, B., Nedorost, N., Maderner, A., Fragner, K., Weissenböck, H., 2011. In situ hybridisation for the detection of Leishmania species in paraffin wax-embedded canine tissues using a digoxigenin-labelled oligonucleotide probe. Vet. Rec. 169, 525

Diniz, S.A., Melo, M.S., Borges, A.M., Bueno, R., Reis, B.P., Tafuri, W.L., Nascimento, E.F. Santos, R.L., 2005. Genital lesions associated with visceral leishmaniasis and shedding of Leishmania sp. in the semen of naturally infected dogs. Vet. Pathol. 42, 650-658.

Dwivedi, D., Toltl, L., Swystun, L., Pogue, J., Liaw, K.-L., Weitz, J., Cook, D., Fox-Robichaud, A., Liaw, P., Group, t.C.C.C.T.B., 2012. Prognostic utility and characterization of cellfree DNA in patients with severe sepsis. Crit. Care 16, R151.

Falangola, M.F., Hanly, A., Galvao-Castro, B., Petito, C.K., 1995. HIV infection of human choroid plexus: a possible mechanism of viral entry into the CNS. J. Neuropathol. Exp. Neurol. 54, 497-503.

Franceschi, A., Merildi, V., Guidi, G., Mancianti, F., 2007. Occurrence of Leishmania DNA in urines of dogs naturally infected with leishmaniasis. Vet. Res. Commun. 31, 335-341.

Gollob, K.J., Antonelli, L.R.V., Faria, D.R., Keesen, T.S.L., Dutra, W.O., 2008. Immunoregulatory mechanisms and CD4-CD8 - (double negative) $\mathrm{T}$ cell subpopulations in human cutaneous leishmaniasis: a balancing act between protection and pathology. Int. Immunopharmacol. 8, 1338-1343.

Gomes, A.A.D., Laurenti, M.D., Ferraro, G.C., de Camargo, M.H.B., Costa, D.C., Machado, G.F. Perri, S.H.V., Marcondes, M., 2012. Subclinical muscle injuries in dogs infected with Leishmania (Leishmania) infantum chagasi. Braz. J. Vet. Pathol. 5, 108-115.

Green, C., Huggett, J.F., Talbot, E., Mwaba, P., Reither, K., Zumla, A.I., 2009. Rapid diagnosis of tuberculosis through the detection of mycobacterial DNA in urine by nucleic acid amplification methods. Lancet Infect. Dis. 9, 505-511.

Hanisch, U.K., Kettenmann, H., 2007. Microglia: active sensor and versatile effector cells in the normal and pathologic brain. Nat. Neurosci. 10, 1387-1394.

Hansen, D.S., Siomos, M.-A., Buckingham, L., Scalzo, A.A., Schofield, L., 2003. Regulation of murine cerebral malaria pathogenesis by CD1d-restricted NKT cells and the natural killer complex. Immunity 18, 391-402.

Hunt, N.H., Ball, H.J., Hansen, A.S., Khaw, L.T., Guo, J., Bakmiwewa, S., Mitchell, A.J., Combes, V., Grau, G.E.R., 2014. Cerebral malaria: gamma-interferon redux. Front. Cell. Infect. Microbiol. 4, 113.

Ikeda, F.A., Laurenti, M.D., Corbett, C.E., Feitosa, M.M., Machado, G.F., Perry, S.H.V., 2007 Histological and immunohistochemical study of the central nervous system of dogs naturally infected by Leishmania (Leishmania) chagasi. Braz. J. Vet. Res. Anim. Sci. 44, 5-11.

Javier, L., Meneses, A.C.O., Rocha, A., Ferreira, M.S., Marquez, J.O., Chapadeiro, E., Lopes, E.R., 1998. Chagasic meningoencephalitis in the immunodeficient. Arq Neuropsiquiatr. 56, 93-97.

Lima, V.M.F., Biazzono, L., Silva, A.C., Correa, A.P.F.L., Luvizotto, M.C.R., 2005. Serological diagnosis of visceral leishmaniasis by an enzyme immunoassay using protein A in naturally infected dogs. Braz. J. Vet. Res. Anim. Sci. 25, 215-218.

Lima, V.M.F., Fattori, K.R., de Souza, F., Eugênio, F.R., Santos, P.S.P., Rozza, D.B., Machado, G.F., 2012. Apoptosis in T lymphocytes from spleen tissue and peripheral blood of $L$. (L.) chagasi naturally infected dogs. Vet. Parasitol. 184, 147-153.

Maeda, S., Ohno, K., Nakashima, K., Fukushima, K., Tsukamoto, A., Suzuki, H., Fujiwara, A. Goto-Koshino, Y., Fujino, Y., Tsujimoto, H., 2012. Molecular cloning and characterization of canine fractalkine and its receptor CX3CR1. Vet. Immunol. Immunopathol. 145, 100-109.

Mantovani, A., 1999. The chemokine system: redundancy for robust outputs. Immunol Today 20, 254-257.

Márquez, M., Pedregosa, J.R., López, J., Marco-Salazar, P., Fondevila, D., Pumarola, M., 2013. Leishmania amastigotes in the central nervous system of a naturally infected dog. J. Vet. Diagn. Investig. 25, 142-146.

Masocha, W., Rottenberg, M.E., Kristensson, K., 2007. Migration of African trypanosomes across the blood-brain barrier. Physiol. Behav. 92, 110-114.

Melo, G.D., Machado, G.F., 2009. Choroid plexus involvement in dogs with spontaneous visceral leishmaniasis: a histopathological investigation. Braz. J. Vet. Pathol. 2, 69-74

Melo, G.D., Machado, G.F., 2011. Glial reactivity in dogs with visceral leishmaniasis: correlation with T lymphocyte infiltration and with cerebrospinal fluid anti-Leishmania antibody titres. Cell Tissue Res. 346, 293-304. 
Melo, G.D., Marcondes, M., Vasconcelos, R.O., Machado, G.F., 2009. Leukocyte entry into the CNS of Leishmania chagasi naturally infected dogs. Vet. Parasitol. 162, 248-256.

Melo, G.D., Seraguci, T.F., Schweigert, A., Silva, J.E.S., Grano, F.G., Peiró, J.R., Lima, V.M.F. Machado, G.F., 2013. Pro-inflammatory cytokines predominate in the brains of dogs with visceral leishmaniasis: a natural model of neuroinflammation during systemic parasitic infection. Vet. Parasitol. 192, 57-66.

Melo, G.D., Silva, J.E.S., Grano, F.G., Homem, C.G., Machado, G.F., 2014. Compartmentalized gene expression of Toll-like receptors 2, 4 and 9 in the brain and peripheral lymphoid organs during canine visceral leishmaniasis. Parasite Immunol. 36, 726-731.

Menezes-Souza, D., Corrêa-Oliveira, R., Guerra-Sá, R., Giunchetti, R.C., Teixeira-Carvalho, A., Martins-Filho, O.A., Oliveira, G.C., Reis, A.B., 2011. Cytokine and transcription factor profiles in the skin of dogs naturally infected by Leishmania (Leishmania) chagasi presenting distinct cutaneous parasite density and clinical status. Vet. Parasitol. 177, 39-49.

Murphy, P.M., 2003. CXC chemokines. In: Henry, H.L., Norman, A.W. (Eds.), Encyclopedia of Hormones. Academic Press, New York, pp. 351-362.

Nascimento, M.S.L., Albuquerque, T.D.R., Do-Valle-Matta, M.A., Caldas, I.S., Diniz, L.F., Talvani, A., Bahia, M.T., Andrade, C.M., Galvão, L.M.C., Câmara, A.C.J., Guedes, P.M.M. 2013. Naturally Leishmania infantum-infected dogs display an overall impairment of chemokine and chemokine receptor expression during visceral leishmaniasis. Vet. Immunol. Immunopathol. 153, 202-208.

Nieto, C.G., Viñuelas, J., Blanco, A., Garcia-Alonso, M., Verdugo, S.G., Navarrete, I., 1996. Detection of Leishmania infantum amastigotes in canine choroid plexus. Vet. Rec. 139, 346-347.

Peeters, D., Peters, I.R., Clercx, C., Day, M.J., 2006. Real-time RT-PCR quantification of mRNA encoding cytokines, CC chemokines and CCR3 in bronchial biopsies from dogs with eosinophilic bronchopneumopathy. Vet. Immunol. Immunopathol. 110, 65-77.

Pfaffl, M.W., Horgan, G.W., Dempfle, L., 2002. Relative expression software tool (RESTC) for group-wise comparison and statistical analysis of relative expression results in real-time PCR. Nucleic Acids Res. 30, e36.

Pritt, B.S., Cooper, K., 2003. The Azzopardi phenomenon. Arch. Pathol. Lab. Med. 127, 1231-1231.

Pumarola, M., Brevik, L., Badiola, J., Vargas, A., Domingo, M., Ferrer, L., 1991. Canine leishmaniasis associated with systemic vasculitis in two dogs. J. Comp. Pathol. 105, 279-286.

Quandt, J., Dorovini-Zis, K., 2004. The beta chemokines CCL4 and CCL5 enhance adhesion of specific CD4 + T cell subsets to human brain endothelial cells. J. Neuropathol. Exp. Neurol. 63, 350-362.

Rabin, R.L., 2003. CC, C, and CX3C chemokines. In: Henry, H.L., Norman, A.W. (Eds.), Encyclopedia of Hormones. Academic Press, New York, pp. 255-263.
Ranasinghe, S., Rogers, M.E., Hamilton, J.G.C., Bates, P.A., Maingon, R.D.C., 2008. A realtime PCR assay to estimate Leishmania chagasi load in its natural sand fly vector Lutzomyia longipalpis. Trans. R. Soc. Trop. Med. Hyg. 102, 875-882.

Reis, A.B., Martins-Filho, O.A., Teixeira-Carvalho, A., Giunchetti, R.C., Carneiro, C.M., Mayrink, W., Tafuri, W.L., Corrêa-Oliveira, R., 2009. Systemic and compartmentalized immune response in canine visceral leishmaniasis. Vet. Immunol. Immunopathol. $128,87-95$.

Rodrigues, A Fighera, R.A., Souza, T.M., Schild, A.L., Barros, C.S.L, 2009. Neuropathology of naturally occurring Trypanosoma evansi infection of horses. Vet. Pathol. 46, 251-258.

São Paulo (Estado), 2006. Superintendência de controle de endemias (SUCEN) e Coordenadoria de controle de doenças (CCD). Secretaria de estado da saúde. Manual de vigilância e controle da leishmaniose visceral americana do estado de São Paulo. A Secretaria, São Paulo (161 pp.).

Solano-Gallego, L., Miro, G., Koutinas, A., Cardoso, L., Pennisi, M., Ferrer, L., Bourdeau, P., Oliva, G., Baneth, G., 2011. LeishVet guidelines for the practical management of canine leishmaniosis. Parasit. Vectors 4, 86.

Strauss-Ayali, D., Baneth, G., Jaffe, C.L., 2007. Splenic immune responses during canine visceral leishmaniasis. Vet. Res. 38, 547-564.

Tafuri, W.L., Santos, R.d.L., Arantes, R.M.E., Gonçalves, R., Melo, M.N., Michalick, M.S.M., Tafuri, W.L., 2004. An alternative immunohistochemical method for detecting Leishmania amastigotes in paraffin-embedded canine tissues. J. Immunol. Methods 292, $17-23$.

Taub, D.D., Conlon, K., Lloyd, A.R., Oppenheim, J.J., Kelvin, D.J., 1993. Preferential migration of activated CD4 + and CD8 + T cells in response to MIP-1 alpha and MIP-1 beta. Science (New York, N.Y.) 260, 355-358.

Viñuelas, J., Garcia-Alonso, M., Ferrando, L., Navarrete, I., Molano, I., Mirón, C., Carcelén, J., Alonso, C., Nieto, C.G., 2001. Meningeal leishmaniosis induced by Leishmania infantum in naturally infected dogs. Vet. Parasitol. 101, 23-27.

Wen, X., Kudo, T., Payne, L, Wang, X., Rodgers, L., Suzuki, Y, 2010. Predominant interferon- $\gamma$-mediated expression of CXCL9, CXCL10, and CCL5 proteins in the brain during chronic infection with Toxoplasma gondii in BALB/c mice resistant to development of toxoplasmic encephalitis. J. Interf. Cytokine Res. 30, 653-660.

Wolburg, H., Paulus, W., 2010. Choroid plexus: biology and pathology. Acta Neuropathol. $119,75-88$

Zhao, L., Toriumi, H., Kuang, Y., Chen, H., Fu, Z.F., 2009. The roles of chemokines in rabies virus infection: overexpression may not always be beneficial. J. Virol. 83, 11808-11818. 